\title{
Impact of phosphorylated insulin-like growth factor-1 receptor on the outcome of breast cancer patients and the prognostic value of its alteration during neoadjuvant chemotherapy
}

\author{
LIANG LI ${ }^{1}$, SHUCHEN LIU ${ }^{1,2}$, LIYUAN LIU ${ }^{1}$, ZHONGBING MA $^{1}$, MAN FENG $^{3}$, CHUNMIAO YE $^{1,2}$, \\ WENZHONG ZHOU ${ }^{1,2}$, YONGJIU WANG ${ }^{1}$, LU LIU ${ }^{1,2}$, FEI WANG ${ }^{1}$, LIXIANG YU ${ }^{1}$, FEI ZHOU ${ }^{1}$, YUJUAN XIANG ${ }^{1}$, \\ SHUYA HUANG ${ }^{1}$, QINYE FU ${ }^{1}$, QIANG ZHANG ${ }^{1}$, DEZONG GAO ${ }^{1}$ and ZHIGANG YU ${ }^{1}$

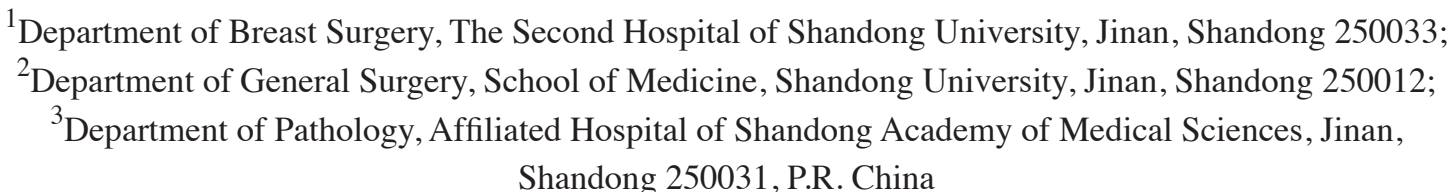

Received November 24, 2017; Accepted April 20, 2018

DOI: $10.3892 /$ etm.2018.6584

\begin{abstract}
The expression of insulin-like growth factor-1 receptor (IGF-1R), which is involved in the genesis and progression of breast cancer, is thought to be associated with the overall survival (OS) of patients. However, the predictive and prognostic significance of the IGF-1R expression in breast cancer remains controversial. The present study aimed to identify the factors associated with the levels of phosphorylated (p)-IGF-1R in breast cancer, their impact on the outcomes of breast cancer patients, and the prognostic value of alterations of $\mathrm{p}-\mathrm{IGF}-1 \mathrm{R}$ during neoadjuvant chemotherapy (NAC). The present study included 348 female breast cancer patients whose paraffin-embedded tumor tissue sections had been collected by biopsy and/or resection, among which the pre-NAC and post-NAC sections were available from 40 patients. Human epidermal growth factor receptor 2 (HER2) positivity and molecular subtype were significantly associated with the presence of $\mathrm{p}-\mathrm{IGF}-1 \mathrm{R}$ in the tumor tissue $(\mathrm{P}<0.05)$. Patients with $\mathrm{p}-\mathrm{IGF}-1 \mathrm{R}$ present in the tumor tissue had a shorter OS ( $\mathrm{P}=0.003)$. The $\mathrm{p}-\mathrm{IGF}-1 \mathrm{R}$ levels in the tumor after NAC differed significantly from those prior to NAC $(\mathrm{P}=0.005)$; however, this alteration in $\mathrm{p}-\mathrm{IGF}-1 \mathrm{R}$ levels was not associated with a shorter OS. In parallel with HER2, p-IGF-1R appears to be a promising indicator for predicting clinical outcomes and may be an attractive target for improving the efficacy of antitumor therapy, particularly
\end{abstract}

Correspondence to: Professor Zhigang Yu, Department of Breast Surgery, The Second Hospital of Shandong University, 247 Zhishan Building, Beiyuan Street, Jinan, Shandong 250033, P.R. China E-mail:yzg@medmail.com.cn

Key words: insulin-like growth factor-1 receptor, breast cancer, prognostic value, neoadjuvant chemotherapy, clinical characteristics, pathological characteristics for patients with HER2-negative, estrogen receptor-positive and luminal B tumors.

\section{Introduction}

Breast cancer is one of the most common malignancies and the leading cause of cancer-associated mortality in females worldwide (1). Considering that breast cancer has a high heterogeneity (2), various specific biomarkers, including estrogen receptor (ER) and human epidermal growth factor-2 (HER2) (3-5), have been discovered to classify breast cancer into four subtypes: Basal-like (BL), luminal A, luminal B and HER2-enriched (6), which allows for the application of more individualized therapies for breast cancer patients. However, the crude mortality due to breast cancer in China in 2010 was 8.65 per 100,000 individuals, and was accountable for $7.90 \%$ of all cancer-associated mortalities in women (7). Therefore, to identify a novel marker consistent with existing ones but able to further refine breast cancer diagnostics has significance for improving the efficacy of breast cancer therapy.

The role of insulin-like growth factor-1 receptor (IGF-1R) in breast carcinogenesis has attracted increasing attention over the last two decades $(8,9)$. IGF-1R is a tyrosine kinase cell surface receptor, which has mitogenic, proliferative and anti-apoptotic effects in cells and was identified to have an increased expression in various types of malignant tumor tissue, including breast cancer (10-13). Several studies have been performed to identify the role and expression of IGF-1R in various subtypes of breast cancer (14). In addition, the overexpression of IGF-1R may be correlated with disease development, aggressive phenotypes, clinical outcomes and the therapy resistance (15). However, the predictive and prognostic significance of IGF-1R expression in breast cancer lesions remains controversial. For instance, certain studies report IGF-1R as a favorable prognostic indicator in breast cancer (16), while others indicate that IGF-1R overexpression is associated with an increased probability of metastasis, a poor response to treatment and a decreased survival rate (17). 
Considering IGF-1R phosphorylation as an essential step for the function of IGF-1R (18), the present study assessed the levels of phosphorylated (p)-IGF-1R in breast cancer tissue, and their correlation with clinicopathological features and clinical outcomes. The present study also investigated whether $\mathrm{p}-\mathrm{IGF}-1 \mathrm{R}$ is a predictive and prognostic biomarker by focusing on its association with the expression of other outcome-associated biomarkers, overall survival (OS) and response to neoadjuvant chemotherapy. It was investigated whether p-IGF-1R has the potential to refine the existing breast cancer classification and prognostication by markers routinely used in the clinic.

\section{Materials and methods}

Patients. A total of 348 female patients admitted to the Department of Breast Surgery of the Second Hospital of Shandong University (Jinan, China) from January 2010 to December 2014 were enrolled in the present study. All patients included in the final analysis were newly diagnosed and histologically confirmed to have breast cancer. The complete clinical data of each patient were recorded, and their breast cancer tissue samples were collected. All records were collected on the basis of patient written informed consent for the use of clinical data, their enrollment in the present study and for the analysis of their tissues. None of them had received any prior cancer treatment, including surgery, chemotherapy, radiotherapy and endocrine therapy. After enrollment, all treatments given by our department were based on NCCN guideline (19). All procedures were in accordance with the guidelines set by the Declaration of Helsinki and approved by the Clinical Research Ethics Committee of the Second Hospital of Shandong University (Jinan, China).

Data collection. The clinical information of all patients was retrieved from electronic and paper-based medical records available from the Second Hospital of Shandong University (Jinan, China), which had been obtained from patients or their family members upon admission. All records were collected on the basis of complete informed consent. The patient data comprised of seven aspects, including i) demographic characteristics: Gender, age, ethnicity, marital status, occupation, height and weight; ii) gynecological details: Age of menarche, duration of menstruation, menstrual cycle and menopausal status; iii) fertility conditions: Number of children, age at first birth and breastfeeding conditions; iv) personal medical history (diagnosed by doctors prior to enrollment in the present study): Hypertension, diabetes mellitus, heart disease, viral hepatitis, benign breast disorders, uterine fibroids and ovarian cysts; v) family history: Breast cancer, other cancer, all types of cancer; vi) physical breast examination: Size, position, texture, mobility and smoothness of tumor and palpation of lymph nodes; vii) treatment-associated information: Time and methods of operation, details regarding lymph node dissection, regimen of chemotherapy, neoadjuvant chemotherapy and endocrine therapy; and viii) pathological characteristics: Pathological types, tumor stage, ER, progesterone receptor (PR), HER2, Ki-67 and lymph node metastasis status.
Immunohistochemistry. In total, immunohistochemical analysis of the expression of p-IGF-1R was performed in 388 breast cancer tissue sections as previously reported $(20,21)$. In brief, all specimens were formalin-fixed and paraffin-embedded. Tissue sections $(3 \mu \mathrm{m})$ were dewaxed with xylene and dehydrated with a graded ethanol series. Antigen retrieval was performed by boiling in citrate buffer (Beyotime Institute of Biotechnology, Haimen, China) and the slides were allowed to cool at room temperature. The slides were then washed with PBS three times for two minutes each time. Next, $50 \mu 1$ of $3 \%$ $\mathrm{H}_{2} \mathrm{O}_{2}$ solution was added to each slide to inhibit the endogenous peroxidase. Slides were incubated with anti-p-IGF-1R polyclonal antibody (cat. no. 39398; 1:200 dilution; Abcam, Cambridge, MA, USA) for $1 \mathrm{~h}$ at $37^{\circ} \mathrm{C}$, followed by washing with PBS three times as above. Secondary antibody (cat. no. DV9000; 1:1 dilution; OriGene Technologies, Inc., Rockville, MD, USA) was added to each slide, followed by incubation at room temperature for $30 \mathrm{~min}$. After washing with PBS, freshly prepared diaminobenzidine solution was added, followed by incubation for $2 \mathrm{~min}$ at room temperature. All slides were washed with tap water and counterstained with hematoxylin for $30 \mathrm{sec}$. Gradient alcohol dehydration and clearing with xylene were performed, and all slides were mounted with neutral balata (OriGene Technologies, Inc., Rockville, MD, USA).

Imaging analysis. Either membranous or cytoplasm staining for $\mathrm{p}$-IGF-1R was defined as positive. The results were assessed by an experienced pathologist using the immunoreactive scoring (IRS) criteria $(22,23)$. A total of 10 randomly selected high-power microscopic fields for each section were observed and 100 cells in each field were counted. IRS=SI (staining intensity) xPP (percentage of positive cells). The staining intensity (22) was rated as 0 (absent), 1 (weak), 2 (moderate) and 3 (strong). The percentage of positive cells was defined as follows: 1, 1-10; 2, 11-50; 3, 51-80; and 4, 81-100\%. For the semi-quantitative analysis, samples with a score of $0-2$, $3 / 4,5-8$ and 9-12 were labeled as,,-+++ and +++ , respectively (23). The sections scoring 0 were considered as negative for $\mathrm{p}$-IGF-1R, and the remaining ones were considered as positive (24).

End-points. OS was defined as the time from diagnosis (date of biopsy) until the time-point of mortality or the last follow-up for patients. Disease-free survival (DFS) was defined as the time from diagnosis (date of biopsy) to events, including local relapse or distant metastases, the occurrence of a new primary tumor or death without evidence of cancer.

Statistical analysis. SPSS version 18.0 (SPSS, Inc., Chicago, IL, USA) was used for statistical analysis of the data. For descriptive analysis, the values of continuous variables were expressed as the mean \pm the standard deviation, and those of categorical variables were presented as the frequency. To describe the distribution of p-IGF-1R and to test whether p-IGF-1R was correlated with any clinicopathological parameters, Pearson's Chi-Square tests were performed for categorical variables. The Wilcoxon rank sum test was performed for comparing $\mathrm{p}$-IGF-1R alterations during neoadjuvant chemotherapy (NAC). Logistic regression was 
performed to identify factors associated with $\mathrm{p}$-IGF-1R levels. Correlations between p-IGF-1R and OS were analyzed by Kaplan-Meier survival analysis and multivariable Cox regression, and differences between subgroups were calculated using the log-rank test. All tests were two-sided, and $\mathrm{P}<0.05$ was considered to indicate a statistically significant difference.

\section{Results}

Patient characteristics. Of the 348 female breast cancer patients enrolled in the present study, 80 received NAC. Among those 80 patients, paraffin-embedded tumor tissue sections from the pre-NAC and post-NAC time-points were available for 40 patients. These tumor tissues had been collected by biopsy and resection, respectively. In total, 388 tumor tissue sections were analyzed by immunohistochemistry to detect p-IGF-1R. The demographic characteristics and medical history of the patients are summarized in Table I.

The age of these 348 patients ranged from 22 to 84 years, and the average age was $51.73 \pm 11.85$ years. The cohort included 144 underweight or normal weight patients $\left(\mathrm{BMI}<24 \mathrm{~kg} / \mathrm{m}^{2}\right.$, accounting for $41.3 \%$ ) and 191 overweight or obese patients (BMI $\geq 24 \mathrm{~kg} / \mathrm{m}^{2}$, accounting for $54.9 \%$ ), while BMI data were missing for 13 patients.

p-IGF-1R levels. Baseline p-IGF-1R was determined by immunohistochemical analysis of p-IGF-1R in tissue collected by resection for patients who did not receive NAC and in the biopsy specimens for patients who were to receive NAC. The baseline expression of p-IGF-1R was assessed in all 348 cases of breast cancer. The results demonstrated that 238 cases $(68.4 \%)$ had a p-IGF-1R negative status, while 110 cases $(31.6 \%)$ had a positive status, among whom weak $(+)$, moderate $(++)$ and strong $(+++)$ p-IGF-1R staining was observed in $74(21.3 \%)$, $32(9.2 \%)$ and $4(1.1 \%)$ cases, respectively. Representative immunohistochemical images are displayed in Fig. 1. The results indicated that compared with the p-IGF-1R-negative cases, less $\mathrm{p}$-IGF-1R positive cases had a history of uterine fibroids ( 2.7 vs. $10.5 \%$; $\mathrm{P}=0.013$; Table I). The distribution of p-IGF-1R among occupations were significantly different $(\mathrm{P}=0.004)$. However, the age, ethnicity, personal medical history regarding hypertension, diabetes, heart disease, breast cancer and benign tumors, and even family history of breast cancer had no significant association with the p-IGF-1R status (P>0.05; Table I).

Clinicopathological characteristics associated with $p$-IGF-1R status. To investigate whether p-IGF-1R may affect the clinicopathological characteristics of breast cancer patients, the association between the p-IGF-1R status and clinicopathological features was assessed (Table II). While 296 patients ( $85.1 \%$ of the total) were diagnosed with invasive ductal carcinoma, 29 (8.3\%), 5 (1.4\%) and 17 (4.9\%) patients were diagnosed with ductal carcinoma in situ, invasive lobular carcinoma and other types of breast cancer, respectively. The results of the statistical analysis indicated that the p-IGF-1R status of breast cancer tissue was neither associated with the pathological type and tumor-nodes-metastasis stage, nor with the status of ER, PR, Ki-67 and lymph node metastasis $(\mathrm{P}>0.05)$. However, the level of $\mathrm{p}-\mathrm{IGF}-1 \mathrm{R}$ in the tumors differed significantly between the groups with and without NAC treatment, and the groups with and without HER2 expression in the tissues. Compared with the p-IGF-1R-negative cases, more p-IGF-1R-positive cases had received NAC (30.0 vs. $19.7 \%$; $\mathrm{P}=0.035$ ). $\mathrm{p}$-IGF-1R-positive cases had a higher rate of positivity for HER2 compared with the p-IGF-1R-negative cases $(30.0$ vs. $14.3 \%)(\mathrm{P}<0.001)$. The difference also reached statistical significance among the groups with different molecular subtypes. More luminal A, luminal $\mathrm{B}$ and triple negative breast cancer (TNBC) tumors had a p-IGF-1R-negative status $(\mathrm{P}=0.022)$. These results suggest that the phosphorylation of IGF-1R may be associated with HER2 signaling.

Downregulation of $p-I G F-1 R$ expression during NAC. To examine whether the level of p-IGF-1R changes during NAC, p-IGF-1R was measured in breast cancer patients prior to and after NAC (Table III). The level of p-IGF-1R was significantly different between the biopsy (pre-NAC) and resection (post-NAC) samples $(\mathrm{P}=0.005)$, and $\mathrm{p}-\mathrm{IGF}-1 \mathrm{R}$ displayed a trend towards a downregulation after NAC.

Factors associated with $p-I G F-1 R$ expression. To examine the association between multiple factors and p-IGF-1R expression in breast cancer, a univariate analysis was performed. The results are presented in Table IV. An analysis of the full dataset indicated that NAC [odds ratio (OR), 1.742; 95\% confidence interval $(\mathrm{CI}), 1.038-2.923 ; \mathrm{P}=0.036]$ and HER2 status (OR, 3.045; 95\% CI, 1.716-5.402; $\mathrm{P}<0.001)$ were significantly associated with the p-IGF-1R levels in breast cancer tissues. Compared to patients with luminal A tumors, patients with the molecular subtype luminal B (OR, 4.067; 95\% CI, 1.001-3.885; $\mathrm{P}=0.050)$ and HER2-enriched subtype (OR, 4.067; 95\% CI, $1.310-12.632 ; \mathrm{P}=0.015)$ had a higher probability of positivity for $\mathrm{p}-\mathrm{IGF}-1 \mathrm{R}$ on immunohistochemistry. According to the multivariate logistic regression (Table V), NAC was significantly associated with p-IGF-1R (OR, 2.326; 95\% CI, 1.018-5.318; $\mathrm{P}=0.045)$. However, the HER2 status was not independently associated with p-IGF-1R (OR, 2.093; 95\% CI, 0.982-4.462; $\mathrm{P}=0.056$ ).

Survival analysis. The follow-up survival data were available for 276 patients. A total of 259 patients survived at the last time-point of follow-up and the mean duration of follow-up was 3 years, ranging from 1-6 years. The mean OS time was 39.8 \pm 11.6 months (range, 10.1-74.7 months). The mean OS time for patients with p-IGF-1R-negative and -positive tumors was $42.0 \pm 10.8$ months and 35.6 \pm 12.0 months, respectively. The p-IGF-1R levels at baseline were significantly associated with OS (log-rank test, $\mathrm{P}=0.003$; Fig. $2 \mathrm{~A}$ ), but not associated with DFS (log-rank test, $\mathrm{P}=0.149$; Fig. 2B). Further Kaplan-Meier analyses were performed, where patients were stratified according to the expression of routinely used biomarkers (Fig. 3). In the subgroups with HER2-negative status $(\mathrm{P}=0.003)$, ER-positive status $(\mathrm{P}=0.027)$ and luminal $\mathrm{B}(\mathrm{P}=0.047)$, patients with $\mathrm{p}$-IGF-1R-positive tumors had a shorter OS (Fig. 3A, D and E, respectively). However, the $\mathrm{p}-\mathrm{IGF}-1 \mathrm{R}$ status had no significant impact on OS in HER2-positive $(\mathrm{P}=0.883)$ and ER-negative patients $(\mathrm{P}=0.187$; 
Table I. Basic demographic characteristics and medical history of the breast cancer patients stratified by p-IGF-1R status .

\begin{tabular}{|c|c|c|c|c|c|}
\hline \multirow[b]{2}{*}{ Variables } & \multirow[b]{2}{*}{$\mathrm{N}(\%)$} & \multicolumn{2}{|c|}{ p-IGF-1R } & \multirow[b]{2}{*}{$\chi^{2}$} & \multirow[b]{2}{*}{ P-value } \\
\hline & & Negative & Positive & & \\
\hline Age (years; range, 22-84) & & & & 2.893 & 0.411 \\
\hline $20-35$ & $24(6.9)$ & $19(8.0)$ & $5(4.5)$ & & \\
\hline $36-50$ & $151(43.4)$ & 99 (41.6) & $52(47.3)$ & & \\
\hline $51-65$ & $132(37.9)$ & $89(37.4)$ & $43(39.1)$ & & \\
\hline$>65$ & $41(11.8)$ & $31(13.0)$ & $10(9.1)$ & & \\
\hline Occupation & & & & 17.101 & 0.004 \\
\hline Farmer & $85(24.4)$ & $53(22.3)$ & $32(29.1)$ & & \\
\hline Worker $^{\mathrm{a}}$ & $85(24.4)$ & $67(28.2)$ & $18(16.4)$ & & \\
\hline Retiree & $62(17.8)$ & $43(18.1)$ & $19(17.3)$ & & \\
\hline Unemployed & $51(14.7)$ & $39(16.4)$ & $12(10.9)$ & & \\
\hline Teacher, public servant, accountant or medical staff & $17(4.9)$ & $13(5.5)$ & $4(3.6)$ & & \\
\hline Other & $48(13.8)$ & $23(9.7)$ & $25(22.7)$ & & \\
\hline Ethnicity & & & & 0.775 & 0.681 \\
\hline Han & 345 (99.1) & $236(99.2)$ & 109 (99.1) & & \\
\hline Hui & $2(0.6)$ & $1(0.4)$ & $1(0.9)$ & & \\
\hline Manchu & $1(0.3)$ & $1(0.4)$ & $0(0)$ & & \\
\hline Marital status & & & & 0.028 & 1.000 \\
\hline Married & $339(97.4)$ & $232(97.5)$ & $107(97.3)$ & & \\
\hline Single & $7(2.0)$ & $5(2.1)$ & $2(1.8)$ & & \\
\hline Unknown & $2(0.6)$ & $1(0.4)$ & $1(0.9)$ & & \\
\hline $\mathrm{BMI}\left(\mathrm{kg} / \mathrm{m}^{2}\right)$ & & & & 1.982 & 0.555 \\
\hline$<18.5$ & $5(1.4)$ & $3(1.3)$ & $2(1.8)$ & & \\
\hline $18.5-23.9$ & $139(39.9)$ & $101(42.4)$ & $38(34.5)$ & & \\
\hline $24.0-27.9$ & $138(39.7)$ & $90(37.8)$ & $48(43.6)$ & & \\
\hline$\geq 28$ & $53(15.2)$ & $36(15.1)$ & $17(15.5)$ & & \\
\hline Unknown & $13(3.7)$ & $8(3.4)$ & $5(4.5)$ & & \\
\hline Family history of breast cancer ${ }^{\mathrm{b}}$ & & & & 0.530 & 0.467 \\
\hline Yes & $31(8.9)$ & $23(9.7)$ & $8(7.3)$ & & \\
\hline No & $317(91.1)$ & $215(90.3)$ & $102(92.7)$ & & \\
\hline Family history of other cancer ${ }^{\mathrm{b}}$ type & & & & 2.178 & 0.140 \\
\hline Yes & $52(14.9)$ & $31(13.0)$ & $21(19.1)$ & & \\
\hline No & $296(85.1)$ & $207(87.0)$ & $89(80.9)$ & & \\
\hline Family history of cancer ${ }^{\mathrm{b}, \mathrm{c}}$ & & & & 0.319 & 0.572 \\
\hline Yes & $82(23.6)$ & $54(22.7)$ & $28(25.5)$ & & \\
\hline No & $266(76.4)$ & $184(77.3)$ & $82(74.5)$ & & \\
\hline Hypertension $^{\mathrm{d}}$ & & & & 2.099 & 0.147 \\
\hline Yes & $80(23.0)$ & $60(25.2)$ & $20(18.2)$ & & \\
\hline No & $268(77.0)$ & $178(74.8)$ & $90(81.8)$ & & \\
\hline Diabetes mellitus $^{\mathrm{d}}$ & & & & 0.915 & 0.339 \\
\hline Yes & $33(9.5)$ & $25(10.5)$ & $8(7.3)$ & & \\
\hline No & $315(90.5)$ & $213(89.5)$ & $102(92.7)$ & & \\
\hline Heart disease ${ }^{\mathrm{d}}$ & & & & 2.117 & 0.146 \\
\hline Yes & $34(9.8)$ & $27(11.3)$ & $7(6.4)$ & & \\
\hline No & $314(90.2)$ & $211(88.7)$ & $103(93.6)$ & & \\
\hline Breast benign tumor $^{\mathrm{d}}$ & & & & 1.322 & 0.361 \\
\hline Yes & $13(3.7)$ & $7(2.9)$ & $6(5.5)$ & & \\
\hline No & $335(96.3)$ & $231(97.1)$ & $104(94.5)$ & & \\
\hline Uterine fibroid $^{\mathrm{d}}$ & & & & 6.150 & 0.013 \\
\hline Yes & $28(8.0)$ & $25(10.5)$ & $3(2.7)$ & & \\
\hline No & $320(92.0)$ & $213(89.5)$ & 107 (97.3) & & \\
\hline
\end{tabular}


Table I. Continued.

p-IGF-1R

\begin{tabular}{lccccc}
\cline { 3 - 3 } Variables & $\mathrm{N}(\%)$ & Negative & Positive & $\chi^{2}$ & P-value \\
\hline Ovarian cyst $^{\mathrm{c}}$ & & & & 0.131 & 0.711 \\
Yes & $8(2.3)$ & $5(2.1)$ & $3(2.7)$ & & \\
No & $340(97.7)$ & $233(97.9)$ & $107(97.3)$ & & \\
\hline
\end{tabular}

Selected parameters refer to ${ }^{\mathrm{a}}$ non-agricultural manual laborer; ${ }^{\mathrm{b}}$ family history; ${ }^{\mathrm{c}}$ all cancer types; ${ }^{\mathrm{d}}$ personal history as diagnosed by doctors prior to enrollment. Values are expressed as n (\%). BMI, body mass index; p-IGF-1R, phosphorylated insulin-like growth factor 1 receptor. Unknown data was not included in statistical analyses.
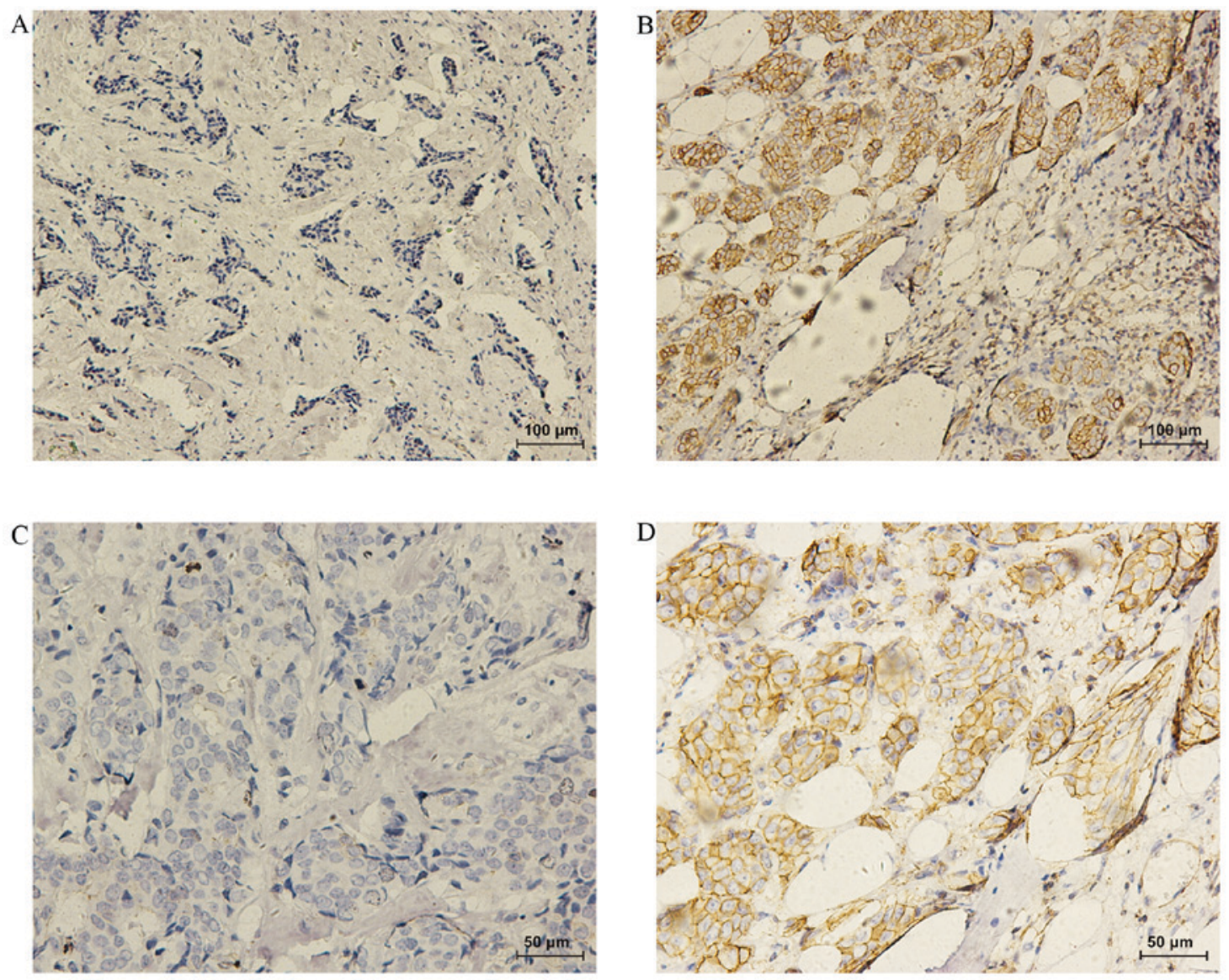

Figure 1. Immunohistochemical analysis of p-IGF-1R in tumor tissues. (A) Representative image of breast cancer tissue with p-IGF-1R-negative status (magnification, x100). (B) Breast cancer tissue with immunostaining for p-IGF-1R (magnification, x100). (C) Breast cancer tissue with p-IGF-1R-negative status (magnification, x200). (D) Breast cancer tissue with immunostaining for p-IGF-1R (magnification, x200). p-IGF-1R, phosphorylated insulin-like growth factor 1 receptor.

Fig. 3B and C, respectively). Cox regression analysis indicated that p-IGF-1R was an independent prognostic factor with OS as the endpoint (OROR, 3.640; 95\% CI, 1.246-10.630; $\mathrm{P}=0.018$; Table VI).

Considering the alteration of p-IGF-1R during NAC mentioned above, the present study further assessed the association of alterations of p-IGF-1R levels with OS and DFS (Fig. 4A and B). Among the 40 patients who received NAC with biopsy and resection data, the follow-up results of only
32 patients were available. The mean follow-up time of the patients was $38.4 \pm 11.9$ months (range, 10.1-58.0 months). The mean OS time for patients in which p-IGF-1R was upregulated, not affected and downregulated after NAC was $40.8 \pm 16.1$, $40.2 \pm 10.9$ and $35.9 \pm 12.5$ months, respectively. This may be due to a small sample size, as OS and DFS were almost the same. Kaplan-Meier analysis did not indicate a statistically significant association between the change of p-IGF-1R after NAC and OS or DFS (log-rank test, $\mathrm{P}=0.273$ or 0.480 , respectively). 
Table II. Association of p-IGF-1R status with various clinicopathological characteristics.

\begin{tabular}{|c|c|c|c|c|c|}
\hline \multirow[b]{2}{*}{ Characteristics } & \multirow[b]{2}{*}{$\mathrm{N}(\%)$} & \multicolumn{2}{|c|}{$\mathrm{p}-\mathrm{IGF}-1 \mathrm{R}$} & \multirow[b]{2}{*}{$\chi^{2}$} & \multirow[b]{2}{*}{ P-value } \\
\hline & & Negative & Positive & & \\
\hline NAC & & & & 4.466 & 0.035 \\
\hline Yes & $80(23.0)$ & 47 (19.7) & $33(30.0)$ & & \\
\hline No & $268(77.0)$ & $191(80.3)$ & $77(70.0)$ & & \\
\hline Pathological type & & & & 0.424 & 0.935 \\
\hline IDC & $296(85.1)$ & $202(84.9)$ & 94 (85.5) & & \\
\hline DCIS & $29(8.3)$ & $20(8.4)$ & $9(8.2)$ & & \\
\hline ILC & $5(1.4)$ & $4(1.7)$ & $1(0.9)$ & & \\
\hline Other type & $17(4.9)$ & $11(4.6)$ & $6(5.5)$ & & \\
\hline Unknown & $1(0.3)$ & $1(0.4)$ & $0(0)$ & & \\
\hline pT stage $\mathrm{e}^{\mathrm{a}}$ & & & & 5.195 & 0.073 \\
\hline $\mathrm{T} 1$ & $131(37.6)$ & $93(39.1)$ & $38(34.5)$ & & \\
\hline $\mathrm{T} 2$ & $99(28.4)$ & $61(25.6)$ & $38(34.5)$ & & \\
\hline $\mathrm{T} 3$ & $6(1.7)$ & $6(2.5)$ & $0(0)$ & & \\
\hline Unknown & $112(32.2)$ & $78(32.8)$ & $34(30.9)$ & & \\
\hline pTNM stage $^{\mathrm{b}}$ & & & & 0.142 & 0.931 \\
\hline $\mathrm{I}$ & $112(32.2)$ & $77(32.4)$ & $35(31.8)$ & & \\
\hline II & $140(40.2)$ & $99(41.6)$ & $41(37.3)$ & & \\
\hline III & $64(18.4)$ & $44(18.5)$ & $20(18.2)$ & & \\
\hline Unknown & $32(9.2)$ & $18(7.6)$ & $14(12.7)$ & & \\
\hline Molecular subtype & & & & 9.598 & 0.022 \\
\hline Luminal A & $60(17.4)$ & 47 (19.9) & $13(11.9)$ & & \\
\hline Luminal B & $204(59.1)$ & $132(55.9)$ & $72(66.1)$ & & \\
\hline HER2-enriched & $17(4.9)$ & $8(3.4)$ & $9(8.3)$ & & \\
\hline TNBC & $23(6.7)$ & $19(8.1)$ & $4(3.7)$ & & \\
\hline Unknown & $41(11.9)$ & 30 (12.7) & $11(10.1)$ & & \\
\hline ER status & & & & 0.005 & 0.945 \\
\hline Positive & $288(82.8)$ & $197(82.8)$ & $91(82.7)$ & & \\
\hline Negative & $53(15.2)$ & $36(15.1)$ & $17(15.5)$ & & \\
\hline Unknown & $7(2.0)$ & $5(2.1)$ & $2(1.8)$ & & \\
\hline PR status & & & & 0.753 & 0.385 \\
\hline Positive & $232(66.7)$ & $162(68.1)$ & $70(63.6)$ & & \\
\hline Negative & $106(30.5)$ & $69(29.0)$ & 37 (33.6) & & \\
\hline Unknown & $10(2.9)$ & $7(2.9)$ & $3(2.7)$ & & \\
\hline HER2 status & & & & 15.173 & $<0.001$ \\
\hline Positive & $67(19.3)$ & $34(14.3)$ & $33(30.0)$ & & \\
\hline Negative & $211(60.6)$ & $160(67.2)$ & $51(46.4)$ & & \\
\hline Unknown & $70(20.1)$ & $44(18.5)$ & $26(23.6)$ & & \\
\hline Ki-67 status & & & & 1.074 & 0.300 \\
\hline $0-14 \%$ & $102(29.3)$ & $73(30.7)$ & $29(26.4)$ & & \\
\hline$>14$ & $228(65.5)$ & $150(63.0)$ & $78(70.9)$ & & \\
\hline Unknown & $18(5.2)$ & $15(6.3)$ & $3(2.7)$ & & \\
\hline Lymph node status ${ }^{\mathrm{c}}$ & & & & 6.364 & 0.095 \\
\hline 0 & $207(59.5)$ & $140(58.8)$ & 67 (60.9) & & \\
\hline $1-3$ & $61(17.5)$ & $48(20.2)$ & $13(11.8)$ & & \\
\hline $4-9$ & $24(6.9)$ & $13(5.5)$ & $11(10.0)$ & & \\
\hline$>9$ & $31(8.9)$ & $24(10.1)$ & $7(6.4)$ & & \\
\hline Unknown & $25(7.2)$ & $13(5.5)$ & $12(10.9)$ & & \\
\hline
\end{tabular}

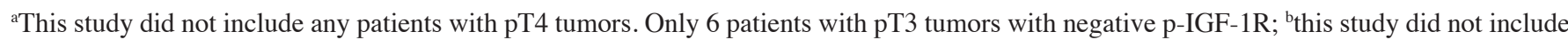
any patients with stage IV tumors; 'Numbers of affected lymph nodes. NAC, neoadjuvant chemotherapy; IDC, invasive ductal carcinoma; DCIS, ductal carcinoma in situ; ILC, invasive lobular carcinoma; ER, estrogen receptor; PR, progesterone receptor; p-IGF-1R, phosphorylated insulin-like growth factor 1 receptor; HER2, human epidermal growth factor receptor 2; pTNM, pathological tumor-nodes-metastasis; TNBC, triple-negative breast cancer. 
Table III. Changes in p-IGF-1R levels in patients receiving NAC.

\begin{tabular}{|c|c|c|c|c|c|c|c|}
\hline \multirow[b]{2}{*}{ Group } & \multirow[b]{2}{*}{$\mathrm{N}(\%)$} & \multicolumn{4}{|c|}{ p-IGF-1R level } & \multirow[b]{2}{*}{$\mathrm{W}$} & \multirow[b]{2}{*}{ P-value } \\
\hline & & 0 & + & ++ & +++ & & \\
\hline Biopsy (pre-NAC) & $40(100)$ & $22(55.0)$ & $12(30.0)$ & $4(10.0)$ & $2(5.0)$ & $1,386.000$ & 0.005 \\
\hline Resection (post-NAC) & $40(100)$ & $34(85.0)$ & $3(7.5)$ & $3(7.5)$ & $0(0)$ & & \\
\hline
\end{tabular}

p-IGF-1R, phosphorylated insulin-like growth factor 1 receptor; NAC, neoadjuvant chemotherapy.

Table IV. Association of clinicopathological characteristics of breast cancer patients with p-IGF-1R positivity determined by univariate logistic regression.

\begin{tabular}{lcc}
\hline & & Overall survival \\
\cline { 2 - 3 } Variables & OR & $95 \%$ CI \\
\hline NAC (yes vs. no) & 1.742 & $1.038-2.923$ \\
pT stage (pT2 and pT3 vs. pT1) & 1.156 & $0.704-1.897$ \\
pT1 & 1.000 & 0.036 \\
pT2 & 1.525 & $0.876-2.652$ \\
pT3 & 0.000 & 0.000 \\
pTNM stage (III/IV vs. I/II) & 0.988 & $0.712-1.372$ \\
Molecular subtype (Luminal B, HER2-enriched and TNBC vs. Luminal A) & 1.085 & $0.788-1.492$ \\
Luminal A & 1.000 & 0.135 \\
Luminal B & 1.972 & $1.001-3.885$ \\
HER2-enriched & 4.067 & $1.310-12.632$ \\
TNBC & 0.761 & $0.220-2.632$ \\
ER (positive vs. negative) & 0.978 & $0.522-1.833$ \\
PR (positive vs. negative) & 0.806 & $0.495-1.313$ \\
HER2 (positive vs. negative) & 3.045 & $1.716-5.402$ \\
Ki-67 ( $\geq 14$ vs. <14\%) & 1.309 & $0.786-2.179$ \\
\hline
\end{tabular}

OR, odds ratio; CI, confidence interval; HER2, human epidermal growth factor receptor 2; TNBC, triple-negative breast cancer; pTNM, pathological tumor-nodes-metastasis; ER, estrogen receptor; PR, progesterone receptor; NAC, neoadjuvant chemotherapy.

\section{Discussion}

In the present study, factors reported by others to be associated with the baseline IGF-1R expression were assessed $(20,21)$. The previously reported induction of p-IGF-1R during NAC and its potential association with OS was also analyzed (25).

IGF-1R is a heterotetrameric transmembrane receptor tyrosine kinase, which is widely expressed in normal human tissues and is frequently upregulated in breast cancer (15). It is well established that IGF-1R is involved in the regulation of numerous biological processes of cells, including differentiation, proliferation, transformation and survival (26), particularly the progression and development of various cancer types (26). The potential mechanism may be that after ligand binding and subsequent phosphorylation, IGF-1R signaling subsequently activates the anti-apoptotic pathways of phosphatidylinositol-3-kinase/AKT and proliferation-driving pathways of Ras/Raf/the mitogen-activated protein kinases (MAPK) extracellular signal-regulated kinases (27). Considering
Table V. Association of the NAC and HER2 status of breast cancer patients with $\mathrm{p}-\mathrm{IGF}-1 \mathrm{R}$ positivity determined by multivariate logistic regression.

\begin{tabular}{lccc}
\hline & \multicolumn{3}{c}{ Overall survival } \\
\cline { 2 - 4 } Variables & OR & $95 \% \mathrm{CI}$ & P-value \\
\hline NAC (yes vs. no) & 2.326 & $1.018-5.318$ & 0.045 \\
HER2 (yes vs. no) & 2.093 & $0.982-4.462$ & 0.056 \\
\hline
\end{tabular}

OR, odds ratio; CI, confidence interval; HER2, human epidermal growth factor receptor 2; NAC, neoadjuvant chemotherapy.

IGF-1R phosphorylation as an essential step for the function of IGF-1R (18), the present study assessed p-IGF-1R in breast cancer tissues. 
A

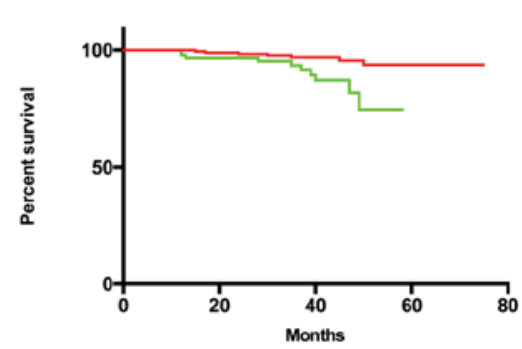

B

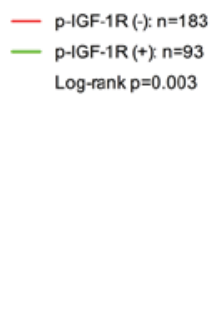

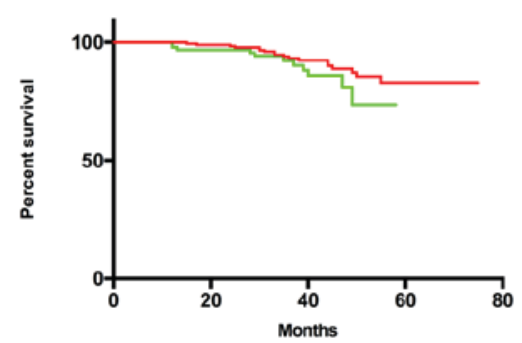

- p-IGF-1R (-): $n=183$

- p-IGF-1R (+): $n=93$ Log-rank $p=0.149$

Figure 2. (A) Overall survival and (B) disease-free survival according to baseline p-IGF-1R levels in tumor tissues. p-IGF-1R, phosphorylated insulin-like growth factor 1 receptor.

A

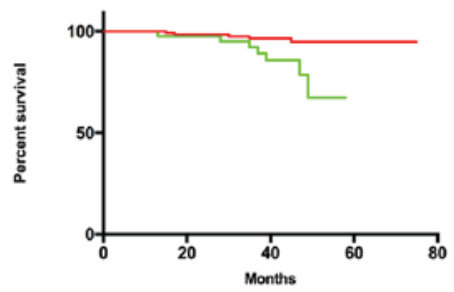

C

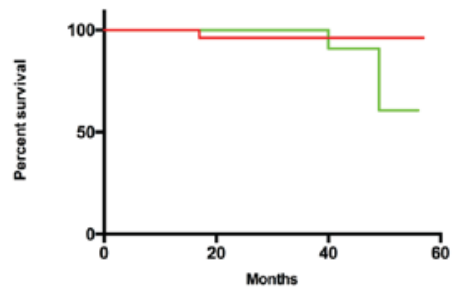

E

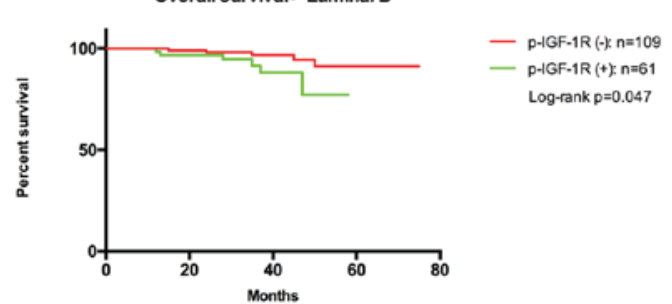

B

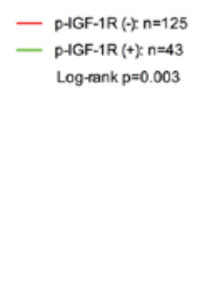
- p-IGF-1R (-) $n=26$ - $p-1 G F-1 R(+)$ n=15 Log-rank $p=0.187$

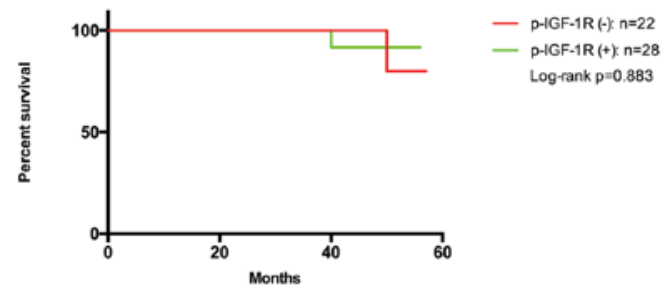

D

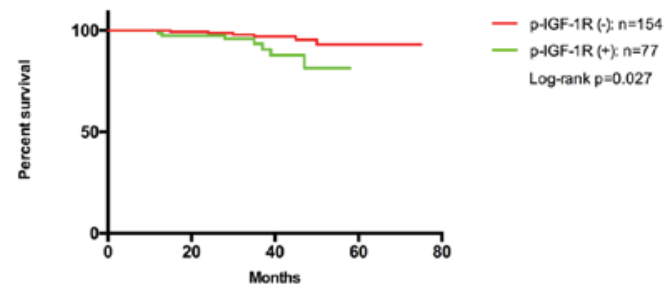

Figure 3. Influence of the expression of various tumor markers in tumor tissues on the overall survival of breast cancer patients stratified by their p-IGF-1R status. Overall survival of breast cancer patients with (A) HER2-negative and (B) HER2-positive status, (C) ER-negative and (D) ER-positive status, and (E) luminal B expression. p-IGF-1R, phosphorylated insulin-like growth factor 1 receptor; ER, estrogen receptor; HER2, human epidermal growth factor receptor 2 .

In the present study, an association between baseline p-IGF-1R expression and a wide range of factors was identified. To date, several studies have investigated the association of clinicopathological features with p-IGF-1R expression in breast cancer $(20,21)$. The present study confirmed that baseline p-IGF-1R levels were significantly associated with NAC, molecular subtypes and the HER2 status according to the univariate analysis, which was in accordance with what was expected. However, the association between p-IGF-1R and HER2 did not reach statistical difference $(\mathrm{P}=0.056)$ based on multivariate logistic regression. It may be speculated that this is due to patients with HER2 (2+) status, which were not further identified by fluorescence in situ hybridization. Furthermore, an insufficient sample size for defining the HER2 status may have led to different statistical results between univariate and multivariate analysis. Even though the multivariate logistic regression result for the HER2 status indicated no statistical significance, integrated univariate analysis indicated that HER2-positive tumors were more likely to express p-IGF-1R than HER2-negative tumors. Although the underlying mechanisms remain elusive, there are three possible explanations for this phenomenon. First, HER 2 may promote aromatase activity by phosphorylating AKT and MAPK (28). Increased aromatase activity leads to elevated estrogen levels, which in turn contributes to the upregulation of IGF-1R (29). Furthermore, elevated IGF-1R levels have been reported to be correlated with trastuzumab resistance in breast cancer (14). Previous studies also demonstrated that the levels of IGF-1R inhibitors, miR-630 and miR-375, are downregulated in trastuzumab-resistant breast cancer, leading to an upregulation of IGF-1R expression $(30,31)$. 
Table VI. Association between p-IGF-1R status and overall survival, as determined by Cox regression analysis.

Overall survival

\begin{tabular}{llcl}
\cline { 2 - 3 } Variable & OR & 95\%CI & P-value \\
\hline IGF-1R (positive vs. negative) & 3.640 & $1.246-10.630$ & 0.018
\end{tabular}

IGF-1R, insulin-like growth factor-1 receptor; OR, odds ratio; CI, confidence interval.

A

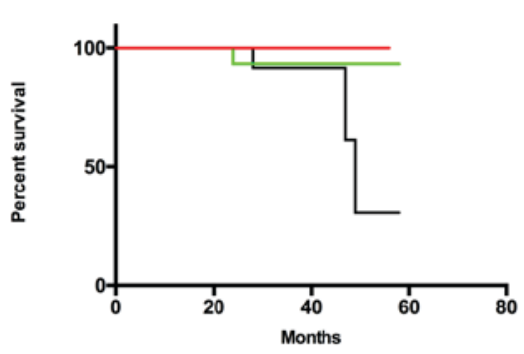

B

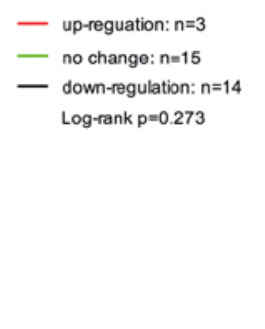

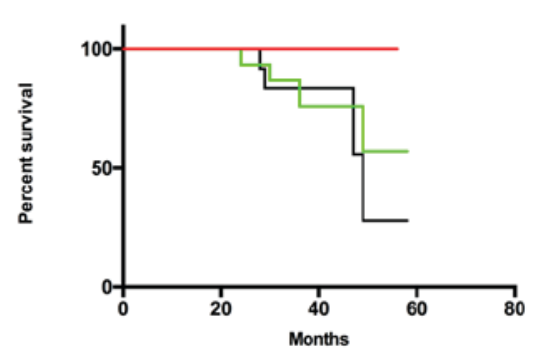

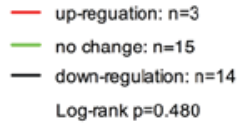

Log-rank $p=0.480$

Figure 4. Effect of alterations in p-IGF-1R expression in the tumor tissues during neo-adjuvant chemotherapy on (A) overall survival and (B) disease-free survival of breast cancer patients. p-IGF-1R, phosphorylated insulin-like growth factor 1 receptor.

Of note, $>60 \%$ of HER2-enriched breast cancer patients with metastases have been reported to present with trastuzumab resistance (32), indicating that HER2-overexpressing tumors are more likely accompanied by IGF-1R positivity. Finally, a heterodimer formed by IGF-1R and HER2 facilitates the process of IGF-1R phosphorylating HER2 and promoting the invasion of tratuzumab-resistant cells (33). Therefore, treatments co-targeting IGF-IR and HER2 have higher antitumor activity than those only targeting either receptor alone (34).

Breast cancer is classified into four major subtypes based on its ER, PR, HER2 and Ki-67 status: Luminal A, luminal B, HER2-enriched and TNBC. One study has demonstrated that high IGF-1R expression levels were more frequently seen in luminal A (52\%), luminal B (57.5\%) and luminal/HER2 (44.8\%) patients, whereas the HER2-enriched (90.3\%) and BL (77.5\%) tumors had lower IGF-1R expression (21). In the present study, it was indicated that the patterns of p-IGF-1R were notably different among several of the above-mentioned subtypes, which means that it may be reasonable to treat breast cancer in different subtypes specifically. Among luminal A, luminal $\mathrm{B}$ and HER2-enriched subgroups, the probability of p-IGF-1R positivity in luminal $\mathrm{B}$ cases is almost twice as high as that in luminal A cases, while the probability in HER2-enriched cases is $>4$ times of that in luminal A cases. TNBC accounted for $\sim 6.7 \%$ of all breast cancer samples assessed in the present study, which is lower than the previously reported rate (10-20\%) (35). More samples are therefore required for future investigation to clarify this discrepancy. Unlike other subtypes, TNBC is a cluster of diseases, with heterogeneity in its genetic locus, intrasubgroup, prognosis and sensitivity to therapy (36), including basal-like 1 (BL1), basal-like 2 (BL2), immunomodulatory (IM), mesenchymal (M), mesenchymal stem-like (MSL), luminal androgen receptor (LAR) and unsTable (UNS), with different characters. BL1 and BL2 are the primary components $(\sim 80 \%)$ of TNBC, characterized by an enrichment of genes, which regulate the cell cycle (36). Higher level of basal cytokeratin can be found in BL1, BL2, UNS and M subtypes while LAR tends to express a high level of luminal cytokeratin. In addition, Masuda et al (37) highlighted that TNBC have different pathological complete response rate (pCR) to standard neoadjuvant chemotherapy. The complexity of pathophenotypes may also be a cause for the undefined association of $\mathrm{p}-\mathrm{IGF}-1 \mathrm{R}$ expression with TNBC in the present study.

At present, the prognostic significance of IGF-1R is controversial. One study reported that IGF-1R expression is not associated with any clinical outcomes (38), while others suggested IGF-1R as a favorable prognostic marker by elucidating that IGF-1R positivity in tumor cells was correlated with DFS, breast-cancer specific survival (BCCS) and OS (21). By contrast, Law et al (39) performed a study including 438 patients with early breast carcinoma and revealed that a high level of p-IGF-1R was significantly correlated with a shorter BCCS. Similarly, the present study indicated that p-IGF-1R positivity predicts a shorter OS, while there was no significance regarding its predictive value for DFS. This suggests that anti-p-IGF-1R targeted therapies may improve the outcome for patients with breast cancer.

In order to further explore the prognostic efficacy of p-IGF-1R in different clinicopathological variables, subgroup analyses were performed. In the subgroups of HER2-negative, ER-positive and luminal B tumors, patients with a p-IGF-1R-positive status had a shorter OS. As is known, unlike that of luminal types, which may be treated with endocrine therapy, the prognosis of TNBC patients is almost always poor due to the lack of molecular treatment targets (21). Patients with TNBC have a high probability of distant metastasis instead of locoregional relapse compared with other types (40). Based on the present results, p-IGF-1R 
positivity may be regarded as an unfavorable prognostic indicator in patients with HER2-negative tumors, and p-IGF-1R may be an attractive therapeutic target, particularly for TNBC.

To date, only a few clinical studies have indicated that chemotherapy may induce alterations in IGF-1R. Heskamp et al (25) demonstrated that upregulation of IGF-1R expression during neoadjuvant therapy predicted poor outcomes in breast cancer patients. These results imply a possible role for IGF-1R in therapy resistance. The association between IGF-1R expression and therapy resistance has been previously observed in several types of cancer. In the present study, changes of p-IGF-1R expression were induced by NAC. However, this alteration was neither associated with OS nor with DFS, which may be due to the small sample size.

In conclusion, the presence of p-IGF-1R determined by immunohistochemistry was indicated to be associated with several clinical and pathological variables, and is paralleled with certain biomarkers linked with poor outcome, including HER2. Based on the Cox regression result, p-IGF-1R is an independent prognostic factor, which indicates that p-IGF-1R may be a promising indicator for predicting clinical outcomes and an attractive target for improving the efficiency of anti-tumor therapy, particularly for patients with HER2-negative, ER-positive and luminal B tumors. Further studies with larger sample sizes of patients receiving NAC will elucidate whether the changes of $\mathrm{p}-\mathrm{IGF}-1 \mathrm{R}$ expression caused by NAC may be associated with survival.

\section{Acknowledgements}

The authors would like to thank Dr Alejandro FernandezEscobar from the Faculty of Medicine of CES University (Medellin, Colombia). for his contribution in revising the manuscript, all patients involved in the present study for their participation and the Department of Pathology (The Second Hospital of Shandong University, Shandong, China) for their collaboration and cooperation.

\section{Funding}

The authors are grateful for the support from the Key Project of the Natural Science Foundation of Shandong Province, China (grant no.ZR2014HZ004), the Key Research and Development Program of Shandong Province (grant no. 2016GSF201130) and the Youth Fund of the Second Hospital of Shandong University (grant no. Y2015010026).

\section{Availability of data and materials}

The datasets used and/or analyzed during the current study are available from the corresponding author on reasonable request.

\section{Authors' contributions}

ZY and LiL conceived and designed the experiments; SL, LYL, ZM, MF, CY, WZ, YW, LuL, FW, LY, FZ, YX, SH, QF, QZ and DG performed the experiments; SL, LYL and ZM analyzed the data; SL and LYL wrote and revised the manuscript; ZY supplied suggestions on study design and prepared the manuscript. The final version of the manuscript has been read and approved by all authors, and each author believes that the manuscript represents honest work.

\section{Ethical approval and consent to participate}

All procedures were in accordance with the guidelines set by the Declaration of Helsinki and approved by the Clinical Research Ethics Committee of the Second Hospital of Shandong University (Jinan, China). All records were collected on the basis of patient written informed consent for the use of clinical data, their enrollment in the present study and for the analysis of their tissues

\section{Patient consent for publication}

Patients consent for publication of the histology images.

\section{Competing interests}

The authors declare that there are no competing interests regarding the publication of this article.

\section{References}

1. Bray F, Ren JS, Masuyer E and Ferlay J: Global estimates of cancer prevalence for 27 sites in the adult population in 2008. Int J Cancer 132: 1133-1145, 2013.

2. Garcia-Closas M, Hall P, Nevanlinna H, Pooley K, Morrison J, Richesson DA, Bojesen SE, Nordestgaard BG, Axelsson CK, Arias JI, et al: Heterogeneity of breast cancer associations with five susceptibility loci by clinical and pathological characteristics. PLoS Genet 4: e1000054, 2008.

3. Gianni L, Dafni U, Gelber RD, Azambuja E, Muehlbauer S, Goldhirsch A, Untch M, Smith I, Baselga J, Jackisch C, et al: Treatment with trastuzumab for 1 year after adjuvant chemotherapy in patients with HER2-positive early breast cancer: A 4-year follow-up of a randomised controlled trial. Lancet Oncol 12: 236-244, 2011.

4. Hu DG, Selth LA, Tarulli GA, Meech R, Wijayakumara D, Chanawong A, Russell R, Caldas C, Robinson JL, Carroll JS, et al: Androgen and estrogen receptors in breast cancer coregulate human UDP-glucuronosyltransferases 2B15 and 2B17. Cancer Res 76: 5881-5893, 2016.

5. Weigel MT and Dowsett M: Current and emerging biomarkers in breast cancer: Prognosis and prediction. Endocr Relat Cancer 17: R245-R262, 2010.

6. Cancer Genome Atlas Network: Comprehensive molecular portraits of human breast tumours. Nature 490: 61-70, 2012.

7. Zeng H, Zheng R, Zhang S, Zou X and Chen W: Female breast cancer statistics of 2010 in China: Estimates based on data from 145 population-based cancer registries. J Thorac Dis 6: 466-470, 2014.

8. Becker MA, Ibrahim YH, Cui X, Lee AV and Yee D: The IGF pathway regulates $E R \alpha$ through a S6K1-dependent mechanism in breast cancer cells. Mol Endocrinol 25: 516-528, 2011.

9. Sarfstein R, Pasmanik-Chor M, Yeheskel A, Edry L, Shomron N, Warman N, Wertheimer E, Maor S, Shochat L and Werner H: Insulin-like growth factor-I receptor (IGF-IR) translocates to nucleus and autoregulates IGF-IR gene expression in breast cancer cells. J Biol Chem 287: 2766-2776, 2012.

10. Giovannucci E: Insulin-like growth factor-I and binding protein-3 and risk of cancer. Horm Res 51 (Suppl 3): S34-S41, 1999.

11. Gong Y, Yao E, Shen R, Goel A, Arcila M, Teruya-Feldstein J, Zakowski MF, Frankel S, Peifer M, Thomas RK, et al: High expression levels of total IGF-1R and sensitivity of NSCLC cells in vitro to an anti-IGF-1R antibody (R1507). PLoS One 4: e7273, 2009.

12. Kucab JE and Dunn SE: Role of IGF-1R in mediating breast cancer invasion and metastasis. Breast Dis 17: 41-47, 2003.

13. Valsecchi ME, McDonald M, Brody JR, Hyslop T, Freydin B, Yeo CJ, Solomides C, Peiper SC and Witkiewicz AK: Epidermal growth factor receptor and insulinlike growth factor 1 receptor expression predict poor survival in pancreatic ductal adenocarcinoma. Cancer 118: 3484-3493, 2012 
14. Farabaugh SM, Boone DN and Lee AV: Role of IGF1R in breast cancer subtypes, stemness, and lineage differentiation. Front Endocrinol (Lausanne) 6: 59, 2015.

15. Christopoulos PF, Msaouel P and Koutsilieris M: The role of the insulin-like growth factor-1 system in breast cancer. Mol Cancer 14: 43, 2015.

16. Papa V, Gliozzo B, Clark GM, McGuire WL, Moore D, Fujita-Yamaguchi Y, Vigneri R, Goldfine ID and Pezzino V: Insulin-like growth factor-I receptors are overexpressed and predict a low risk in human breast cancer. Cancer Res 53: 3736-3740, 1993

17. Bahhnassy A,Mohanad M,Shaarawy S, Ismail MF,El-Bastawisy A, Ashmawy AM and Zekri AR: Transforming growth factor- $\beta$, insulin-like growth factor I/insulin-like growth factor I receptor and vascular endothelial growth factor-A: Prognostic and predictive markers in triple-negative and non-triple-negative breast cancer. Mol Med Rep 12: 851-864, 2015.

18. Kelly GM, Buckley DA, Kiely PA, Adams DR and O'Connor R: Serine phosphorylation of the insulin-like growth factor I (IGF-1) receptor C-terminal tail restrains kinase activity and cell growth. J Biol Chem 287: 28180-28194, 2012.

19. Gradishar WJ, Anderson BO, Balassanian R, Blair SL, Burstein HJ, Cyr A, Elias AD, Farrar WB, Forero A, Giordano SH, et al: Breast cancer version 2.2015. J Natl Compr Canc Netw 13: 448-475, 2015.

20. Hartog H, Horlings HM, van der Vegt B, Kreike B, Ajouaou A, van de Vijver MJ, Marike Boezen $\mathrm{H}$, de Bock GH, van der Graaf WT and Wesseling J: Divergent effects of insulin-like growth factor-1 receptor expression on prognosis of estrogen receptor positive versus triple negative invasive ductal breast carcinoma. Breast Cancer Res Treat 129: 725-736, 2011.

21. Yerushalmi R, Gelmon KA, Leung S, Gao D, Cheang M, Pollak M, Turashvili G, Gilks BC and Kennecke H: Insulin-like growth factor receptor (IGF-1R) in breast cancer subtypes. Breast Cancer Res Treat 132: 131-142, 2012.

22. Remmele W and Stegner HE: Recommendation for uniform definition of an immunoreactive score (IRS) for immunohistochemical estrogen receptor detection (ER-ICA) in breast cancer tissue. Pathologe 8: 138-140, 1987 (In German).

23. Friedrichs K, Gluba S, Eidtmann $\mathrm{H}$ and Jonat W: Overexpression of p53 and prognosis in breast cancer. Cancer 72: 3641-3647, 1993

24. Zhao S, Chen SS, Gu Y, Jiang EZ and Yu ZH: Expression and clinical significance of sushi domain-containing protein 3 (SUSD3) and insulin-like growth factor-I receptor (IGF-IR) in breast cancer. Asian Pac J Cancer Prev 16: 8633-8636, 2015.

25. Heskamp S, Boerman OC, Molkenboer-Kuenen JD, Wauters CA Strobbe LJ, Mandigers CM, Bult P, Oyen WJ, van der Graaf WT and van Laarhoven HW: Upregulation of IGF-1R expression during neoadjuvant therapy predicts poor outcome in breast cancer patients. PLoS One 10: e0117745, 2015.

26. Mauro L, Naimo GD, Ricchio E, Panno ML and Andò S: Cross-Talk between adiponectin and IGF-IR in breast cancer. Front Oncol 5: 157, 2015.

27. Surmacz E: Growth factor receptors as therapeutic targets: Strategies to inhibit the insulin-like growth factor I receptor Oncogene 22: 6589-6597, 2003.

28. Su B, Wong C, Hong Y and Chen S: Growth factor signaling enhances aromatase activity of breast cancer cells via post-transcriptional mechanisms. J Steroid Biochem Mol Biol 123: 101-108, 2011.
29. Casa AJ, Potter AS, Malik S, Lazard Z, Kuiatse I, Kim HT, Tsimelzon A, Creighton CJ, Hilsenbeck SG, Brown PH, et al: Estrogen and insulin-like growth factor-I (IGF-I) independently down-regulate critical repressors of breast cancer growth. Breast Cancer Res Treat 132: 61-73, 2012.

30. Corcoran C, Rani S, Breslin S, Gogarty M, Ghobrial IM, Crown J and O'Driscoll L: miR-630 targets IGF1R to regulate response to HER-targeting drugs and overall cancer cell progression in HER2 over-expressing breast cancer. Mol Cancer 13: 71, 2014.

31. Ye XM, Zhu HY, Bai WD, Wang T, Wang L, Chen Y, Yang AG and Jia LT: Epigenetic silencing of miR-375 induces trastuzumab resistance in HER2-positive breast cancer by targeting IGF1R. BMC Cancer 14: 134, 2014

32. Vogel CL, Cobleigh MA, Tripathy D, Gutheil JC, Harris LN, Fehrenbacher L, Slamon DJ, Murphy M, Novotny WF, Burchmore M, et al: Efficacy and safety of trastuzumab as a single agent in first-line treatment of HER2-overexpressing metastatic breast cancer. J Clin Oncol 20: 719-726, 2002.

33. Nahta R, Yuan LX, Zhang B, Kobayashi R and Esteva FJ: Insulin-like growth factor-I receptor/human epidermal growth factor receptor 2 heterodimerization contributes to trastuzumab resistance of breast cancer cells. Cancer Res 65: 11118-11128, 2005.

34. Chen C, Zhang Y, Zhang Y, Li J, Tsao SW and Zhang MY: Superior antitumor activity of a novel bispecific antibody cotargeting human epidermal growth factor receptor 2 and type I insulin-like growth factor receptor. Mol Cancer Ther 13: 90-100, 2014.

35. Papa A, Caruso D, Tomao S, Rossi L, Zaccarelli E and Tomao F: Triple-negative breast cancer: Investigating potential molecular therapeutic target. Expert Opin Ther Targets 19: 55-75, 2015.

36. Mancini P, Angeloni A, Risi E, Orsi E and Mezi S: Standard of care and promising new agents for triple negative metastatic breast cancer. Cancers (Basel) 6: 2187-2223, 2014.

37. MasudaH,Baggerly KA,Wang Y,Zhang Y,Gonzalez-Angulo AM, Meric-Bernstam F, Valero V, Lehmann BD, Pietenpol JA, Hortobagyi GN, et al: Differential response to neoadjuvant chemotherapy among 7 triple-negative breast cancer molecular subtypes. Clin Cancer Res 19: 5533-5540, 2013.

38. Shimizu C, Hasegawa T, Tani Y, Takahashi F, Takeuchi M, Watanabe T, Ando M, Katsumata N and Fujiwara Y: Expression of insulin-like growth factor 1 receptor in primary breast cancer: Immunohistochemical analysis. Hum Pathol 35: 1537-1542, 2004.

39. Law JH, Habibi G, Hu K, Masoudi H, Wang MY, Stratford AL, Park E, Gee JM, Finlay P, Jones HE, et al: Phosphorylated insulin-like growth factor-i/insulin receptor is present in all breast cancer subtypes and is related to poor survival. Cancer Res 68: 10238-10246, 2008

40. Haffty BG, Yang Q, Reiss M, Kearney T, Higgins SA, Weidhaas J, Harris L, Hait W and Toppmeyer D: Locoregional relapse and distant metastasis in conservatively managed triple negative early-stage breast cancer. J Clin Oncol 24: 5652-5657, 2006.

This work is licensed under a Creative Commons Attribution-NonCommercial-NoDerivatives 4.0 International (CC BY-NC-ND 4.0) License. 